\title{
Relativistic cross sections of tidal disruption events
}

\author{
P.B. Ivanov \\ Astro Space Centre, PN Lebedev Physical Institute, 84/32 Profsoyuznaya st., Moscow, 117997, Russia
}

\begin{abstract}
In this contribution I introduce and discuss the so-called cross sections of tidal disruption and partial mass stripping of stars after a tidal encounter with a rotating supermassive black hole. The presented approach is fully relativistic. Possible applications and generalisations of the discussed results are also shortly reviewed.
\end{abstract}

\section{INTRODUCTION}

In the simplest Newtonian formulation of the tidal disruption problem it takes place when the periastron of the stellar orbit is smaller than the so-called Roche tidal radius

$$
r_{T}=\sqrt[3]{\frac{M}{m}} R_{s t} \approx 1.5 \cdot 10^{13} \sqrt[3]{\frac{M_{7}}{m_{*}}} R_{*} \mathrm{~cm},
$$

where $M$ is the black hole mass and $m$ is the mass of the star, $R_{s t}$ is the stellar radius, $M_{7}=M / 10^{7} M_{\odot}, m_{*}=m / M_{\odot}$ and $R_{*}=R_{s t} / R_{\odot}$, see e.g. [7]. Since the semi-major axis of the stellar orbit can be as large as a few $p c$, tidally disrupted stars have highly elongated (technically, parabolic) orbits. Another important process that competes with that of the tidal disruption is the relativistic process of direct capture of stars by the black hole. The star can be directly captured by the black hole when its periastron is of the order of the black hole gravitational radius

$$
r_{g}=\frac{2 G M}{c^{2}} \approx 3 \cdot 10^{12} M_{7} c m
$$

Since for a black hole with mass larger than $10^{7} M_{\odot}$ the ratio $r_{g} / r_{T}>0.2$, the relativistic treatment of the process of the tidal disruption is important. Also, the simple approach outlined above cannot describe more complicated effects, such as partial mass stripping of the star, distribution of different quantities, e.g. orbital parameters of the gas lost by the star over orbital parameters of the stellar orbit before the tidal disruption event, etc.

In a more advanced formulation of the problem different numerical approaches are used. Typically, they are based either on finite difference or on SPH methods, see, for example, other contributions to the proceedings of this conference and references therein. They are suited quite well to describe the process in detail, for a number of chosen orbital parameters of the star. However, even the state-of-the-art computational facilities cannot be used to survey the whole parameter space of the problem, which is, in general, a three-dimensional non-stationary problem of relativistic hydrodynamics. Therefore, in order to do it, faster numerical schemes have to be employed.

Here I would like to describe briefly such a scheme, which was developed in the papers [8] and [9] and used

a e-mail: pbi20@cam.ac.uk by [10] to calculate the so-called cross sections of tidal disruption and mass loss from the star after its flyby around a supermassive black hole (see also [2]). These cross sections allow us to describe the process of mass stripping from the star by a black hole for practically all interesting orbital parameters, black hole masses and spins, for a given model of unperturbed star. It will be assumed later that the star is modelled as a $\mathrm{n}=1.5$ polytrope.

The cross sections will be discussed below as well as possible applications and future development of this approach.

\section{BASIC DEFINITIONS AND FORMULATION OF THE PROBLEM IN GENERAL RELATIVITY}

\subsection{Orbital parameter and definition of cross sections}

Roughly, the cross sections of tidal disruption define the section of a bunch of trajectories of stars falling onto a rotating black hole with a given inclination angle $\theta_{\infty}$ with respect to the black hole rotational axis where the stars are tidally disrupted. This is analogous to the well-known cross section of direct capture of stars by the black hole. In the same way I define cross sections of partial mass stripping as sections of the bunch of trajectories wherein stars lose a certain percentage of mass after a flyby around the black hole.

In order to define these cross sections in a quantitative way one can consider the stellar trajectory far from black hole, where all components of angular momentum are approximately conserved as well as the inclination angle $\theta_{\infty}$, and introduce two components of orbital angular momentum vector (per unit of mass) $j_{\phi}$ and $j_{\theta}$ projected onto the azimuthal direction and the direction of increasing of the co-latitude $\theta$, respectively ${ }^{1}$. For a given inclination angle we can characterise stellar orbits considering the plane $\left(j_{\phi}, j_{\theta}\right)$. Defining on this plane regions corresponding to orbits where stars are either captured by the black hole or tidally disrupted/partially stripped we can introduce the corresponding cross sections of direct capture, tidal disruption and partial mass loss, respectively. Taking into account that the problem is symmetric with respect to the reflection $j_{\phi} \rightarrow-j_{\phi}$, we can consider only positive values of $j_{\phi}>0$.

\footnotetext{
${ }^{1}$ Let the velocity components at the orbit apastron be $v_{\phi}$ and $v_{\theta}$ and the apastron distance be $r_{a}$. Then, $j_{\phi}=r_{a} v_{\theta}$ and $j_{\theta}=r_{a} v_{\phi}$.
} 
Alternatively, one can characterise the stellar orbit by the projection of the specific angular momentum onto the axis of rotation of the black hole, $L_{z}$ and the square of the projection of the angular momentum onto the equatorial plane, $Q$. It is easy to show that the sodefined integral of motion $Q$ coincides with the wellknown Carter integral when the gravitational field of the black hole dominates over the gravitational field of the central stellar cluster. The quantities $L_{z}$ and $Q$ are related to $j_{\phi}$ and $j_{\theta}$ as

$$
L_{z}=j_{\theta} \sin \theta_{\infty}, \quad Q=j_{\phi}^{2}+j_{\theta}^{2} \cos ^{2} \theta_{\infty} .
$$

The Carter integral $Q$ is always positive for parabolic orbits, and we will use its square root $q=\sqrt{Q}$ to have the same dimensions for the integrals of motion $q$ and $L_{z}, j_{\phi}$, $j_{\theta}$. For $\theta_{\infty}=\pi / 2$ and $j_{\phi}>0, L_{z}=j_{\theta}$ and $q=j_{\phi}$.

The black hole itself is characterised by only two parameters, its mass $M$ and the rotational parameter $a=c L_{h} /\left(G M^{2}\right)<1$, where $L_{h}$ is the black hole angular momentum.

We use $\tilde{L}_{z}=L_{z} /\left(c r_{g}\right), \tilde{q}=q /\left(c r_{g}\right), \tilde{j}_{\phi}=j_{\phi} /\left(c r_{g}\right)$ and $\tilde{j}_{\theta}=j_{\theta} /\left(c r_{g}\right)$. We do not write tilde below assuming that all quantities mentioned above are dimensionless. The black hole masses are expressed in terms of $10^{7} M_{\odot}$ in Figures below and masses of stars in units of the solar mass.

\subsection{The model of tidally disrupted star}

Numerical calculations of the tidal disruption cross sections were performed using the model of tidally disrupted star formulated and studied in [8] and [9]. In this model the star is assumed to consist of enclosed elliptical shells with different parameters and different orientations with respect to a Cartesian coordinate system centred at the centre of mass of the star. Equations of motion follow from the so-called virial relations written for a particular shell. The model is a generalisation of the so-called affine model of Luminet and Carter (see e.g. [3], [4]). However, unlike the affine model our approach allows one to consider nonself-similar motions of the shells, and, hence, can be used to describe effects such as the partial mass loss by the star after its flyby around black hole.

The model is effectively a one-dimensional Lagrangian model. Therefore, it can be evolved numerically roughly $10^{3}$ times faster than more complicated finite-difference or SPH three-dimensional hydrodynamical schemes. It demonstrates rather good agreement with the hydrodynamical schemes, see, for example, comparison of the model with recent three dimensional calculations in [6], their Fig. 3.

\section{THE CROSS SECTIONS OF TIDAL DISRUPTION}

The cross sections of tidal disruption and mass stripping are described in Figures 1-4. In Figures 1 and 2 I show the cross sections on the plane $\left(L_{z}, q\right)$ for orbits having the inclination $\Theta_{\infty}=\pi / 2$, for a nearly maximal black hole rotation with the rotational parameter $a=0.999$. Curves of
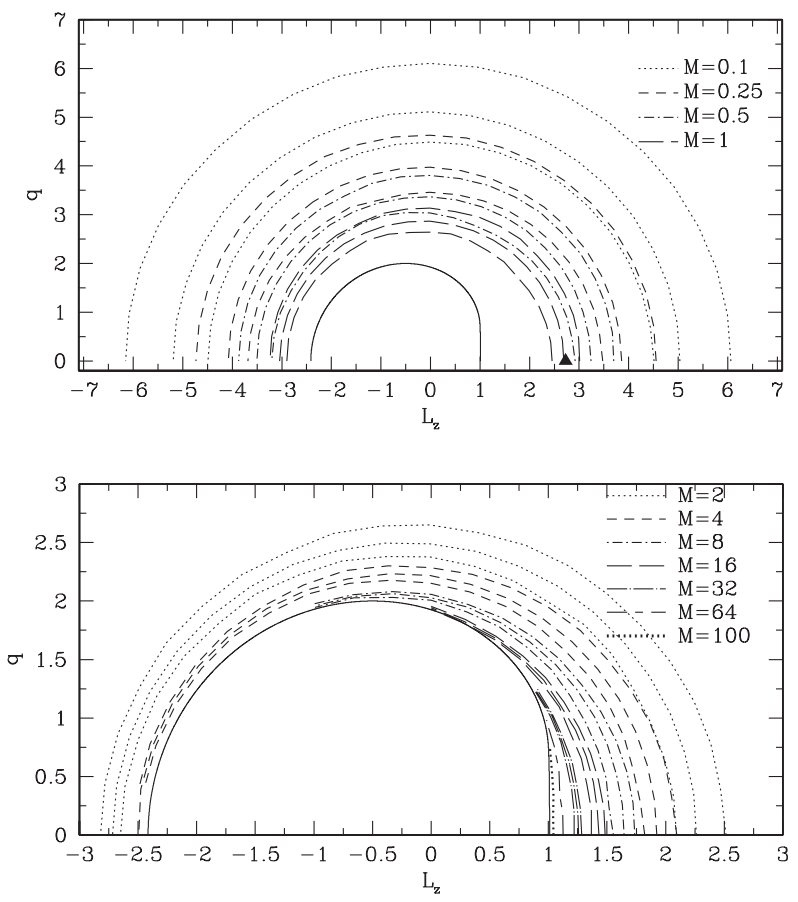

Figure 1. The calculated cross sections of tidal disruption for the case $a=0.999$ and different black hole masses. The levels of the amount of mass lost by the star, $M_{\text {lost }}$, are shown by curves of different types corresponding to different masses of the black hole. Three curves of the same type show $M_{\text {lost }}=0.1$ (the outer curve), $M_{\text {lost }}=0.5$ (the middle curve) and $M_{\text {lost }}=1$ (the inner curve). Note that the curves corresponding to the different black hole masses may overlap (e.g. $M_{\text {lost }}(M=0.1)=1$ and $M_{\text {lost }}(M=$ $0.25)=0.1)$. The solid curve shows the cross section of direct capture. Top: The case of small black hole masses is shown, $M=0.1,0.25,0.5$ and 1 . The triangle shows the result of 3D finite difference calculations of [5]. They obtained $M_{\text {lost }}^{D} \sim 0.5$. Bottom: The case of large black hole masses, $M=2,4,8,16$, 32,64 and 100. Note that in the case $M=64$ and $M=100$ the curves corresponding to different $M_{\text {lost }}$ almost coincide, and we show only the curves corresponding to $M_{\text {lost }}=1$.

different types correspond to different black hole masses (in units $10^{7} M_{\odot}$ ). For a given mass, the outer, intermediate and inner curves of the same type give the amount of mass lost after the flyby $M_{\text {lost }}=0.1, M_{\text {lost }}=0.5$ and $M_{\text {lost }}=1$, respectively. Thus, the inner curve corresponds to full disruption of the star and marks the boundary of the tidal disruption cross section. The solid curve shows the boundary of the cross section of direct capture of stars by the black hole. Let me remind that I express $q$ and $L_{z}$ in units $G M / c$. Being expressed in these units the boundary of the cross section of direct capture does not depend on $M$ while a typical size of the tidal disruption/mass stripping cross sections decreases $\propto M^{-1 / 3}$. When $M>10^{7} M_{\odot}$ the size of these cross sections becomes comparable to the size of the direct capture cross sections and when $M>10^{9}$ the stars of solar mass and radius can only be swallowed undisrupted by the black hole. Similarly to the direct capture cross sections the ones corresponding to the tidal disruption are shifted towards negative values of $L_{z}$. However, their shift is smaller than that of the direct capture cross section. Therefore, when they happen to 

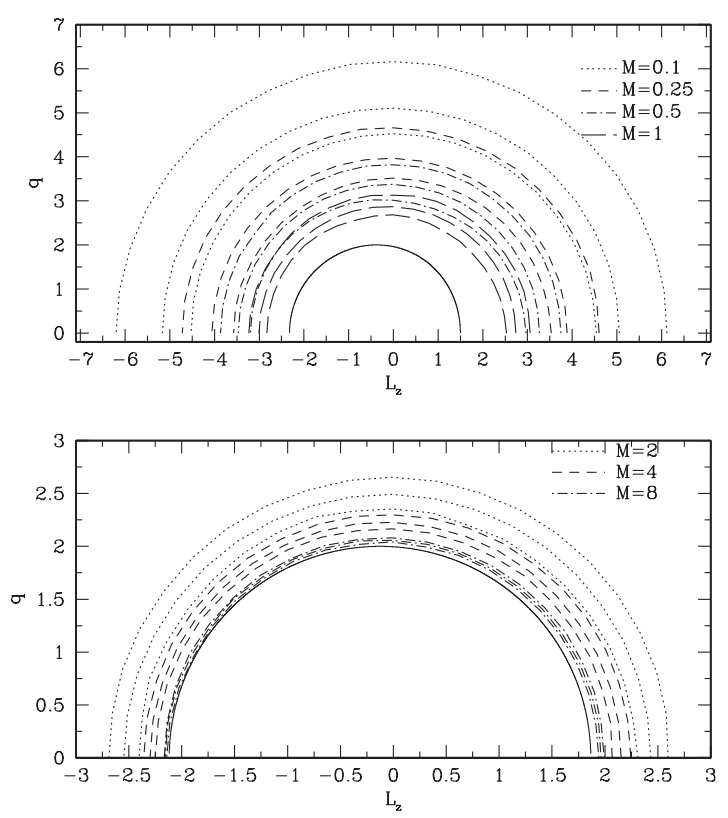

Figure 2. Same as 1 but for $a=0.25$.

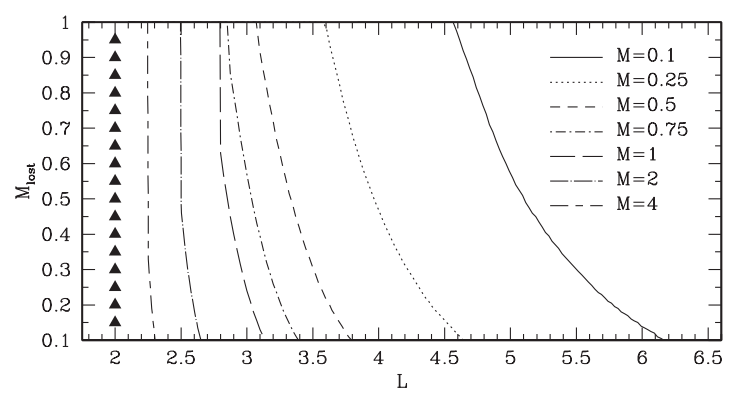

Figure 3. The amount of mass lost by the star calculated for the case $a=0$. Since the cross sections in this case are concentric circles in the plane $\left(L_{z}, q\right)$ we show $M_{\text {lost }}$ as a function of the radius $L=\sqrt{q^{2}+L_{z}^{2}}$ for the different black hole masses $M=$ $0.1,0.25,0.5,0.75,1,2$ and 4 . Vertical triangles show the position of the radius of the capture cross section $L_{\text {capt }}=2$.

intersect each other (roughly, when $M>4 \cdot 10^{7} M_{\odot}$ in the model we use) predominantly stars with positive values of $L_{z}$ are disrupted by the black hole.

In Fig. 2 I show the same results but for the case of $a=0.25$. As seen from this Fig. the behaviour of the cross sections is similar to the previous case but effects of rotation are less prominent.

When $a=0$ the cross sections are concentric circles on the plane $\left(L_{z}, q\right)$ and we can show $M_{\text {lost }}$ as a function of $L=\sqrt{L_{z}^{2}+q^{2}}$, see Fig. 3. As seen from this Fig. the region of partial mass loss is almost comparable to the size of the tidal disruption cross section for a relatively low mass black hole with $M=10^{6} M_{\odot}$ while for black holes with larger masses the relative size of this region shrinks.

In Fig. 4 I show results describing dependence of the cross sections on the angle $\Theta_{\infty}$. As can be deduced from equation (3), for given values of $L_{z}$ and $q$ the angle $\Theta_{\infty}$ must be in the range

$$
\Theta_{-} \leq \Theta_{\infty} \leq \Theta_{+}
$$

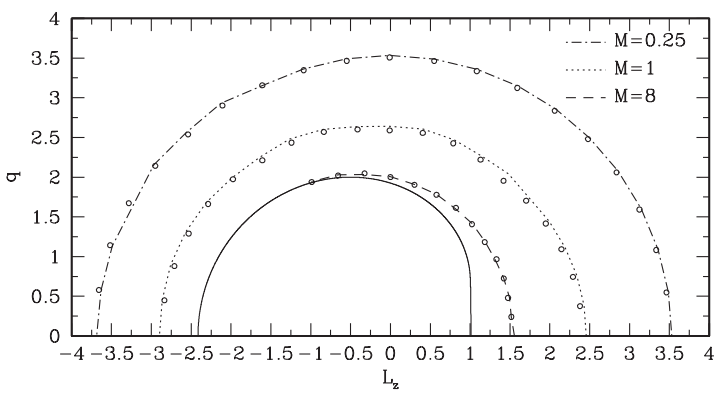

Figure 4. The tidal cross sections calculated for $\Theta_{\infty}=\Theta_{+}$ (shown by the circles) in comparison to the tidal cross sections calculated for $\Theta_{\infty}=\pi / 2$ for $a=0.9999$ and $M_{\text {lost }}=1$. Note that the case $\Theta_{\infty}=\Theta_{-}$is equivalent to the case shown, by symmetry.

where

$$
\Theta_{ \pm}=\pi / 2 \pm \arctan \left|\frac{q}{L_{z}}\right|
$$

In Fig. $4 \mathrm{I}$ present the results of calculation of $M_{\text {lost }}$ for $\Theta_{\infty}=\Theta_{+}$in comparison with the case $\Theta_{\infty}=\pi / 2$. As seen from this Fig. there is almost no dependence on this angle. This may explain why even a simpler approach to construction of the tidal disruption cross section based on some criterion of tidal disruption at periastron of the stellar orbit ([2], [11]), where the dependence on $\Theta_{\infty}$ is absent, gives results which are in qualitative agreement with what is reported here. Note that this degeneracy should be confirmed by the 3D methods. If confirmed it greatly simplifies construction of the cross sections since it reduces the number of dimensions of the parameter space of the problem. There are even more radical means to reduce the dimensionality of the parameter space. Namely, it can be seen that the levels of constant $M_{\text {lost }}$ are, in fact, approximate circles on the plane $\left(L_{z}, q\right)$ shifted in the direction of negative $L_{z}$. Thus, our model suggests that for given values of $M, a$ and $M_{\text {lost }}$ it suffices to calculate numerically only radius of this circle and the value of the shift, see [10] for explicit dependencies of these quantities on $M$ and $a$. Let me stress, however, that this result must be checked with the modern $3 \mathrm{D}$ hydrodynamical schemes as well.

\section{DISCUSSION}

Let me discuss possible implications and generalisations of the reported results. 1) In the standard loss cone theory of interaction of stars in central stellar clusters with a supermassive black hole in galactic nuclei it is typically assumed that stars can change their angular momenta as a result of two-body gravitational scattering, which leads to diffusive wandering thereof in two-dimensional angular momentum space. The stars having their angular momenta inside the loss cone, which may be defined as the union of tidal disruption and direct capture cross section, are removed from the cluster. There are two possible regimes: the so-called full and empty loss cone regimes depending on whether a typical change of angular momentum due to two-body processes is larger or smaller than a typical size of the loss cone, respectively. Both regimes can be present in the same cluster with full 
(empty) loss cone regime operating for stars having larger (smaller) orbital periods. It is normally assumed that the loss cone is symmetric with respect to the origin in the angular momentum space. As follows from the discussion above black hole rotation breaks this symmetry. Therefore, it could induce a degree of anisotropy in distribution of stars over angular momenta, which may have some observational consequences. Thus, in my opinion, it is important to take into account this effect in studies of stellar dynamics in central clusters. 2) There is a region in the angular momentum space where tidal interactions lead to the partial stripping of mass from stars and some gravitationally bound remainders are left after the tidal encounters. These stellar remainders should also be strongly perturbed after the flyby around the black hole and spun up to high rotation frequencies. Provided that two-body interactions scatter such remainders back into 'safe' regions of the angular momentum space they could relax to the ordinary state for a long time, say, of the order of their thermal time scale. Observations of such objects could manifest dynamical tidal interactions with the black hole, see, for example, [1]. Clearly, the results reported here can be used to calculate the rates of such processes. These effects may occur most probably in the regime of the full loss cone. Alternatively, in the empty loss cone regime a star may experience a number of 'grazing' encounters with the black hole losing a small fraction of mass during every encounter. This could lead to a recurring activity in galactic centres, see a discussion of a similar effect of tidal interaction of a white dwarf with an intermediate mass black hole in [12]. 3) I have considered only 'the first passage' problem assuming that the star is in its unperturbed state before the tidal encounter. It is important to generalise this approach to the case of multiple tidal encounters calculating the corresponding cross section for stars, which have been already perturbed by the black hole in the course of previous periastron passages. 4) As I have stressed above it is quite important to check the results reported here with $3 \mathrm{D}$ hydrodynamical simulations. It is especially important to verify whether the obtained degeneracies of the tidal cross sections are present in more realistic treatments of the problem. 5) In a similar way, one can introduce other cross sections characterising tidal encounters, say, mapping quantities determining distribution of gas unbound after a tidal encounter on the plane $\left(L_{z}, q\right)$. Also, it is straightforward to generalise the results to other stellar models.

I am grateful to my collaborators on this project. It is sincere pleasure to thank many participants of this workshop for very interesting discussions and the organisers of this meeting for providing a very vivid and invigorating atmosphere.

The work was supported in part by the Dynasty foundation, by the programme 22 of the presidium of RAS, by grant no. NSh 2915.2012.2 from the President of Russia and by Federal program "Scientific personnel" contract 8422.

\section{References}

[1] Alexander, T., Livio, M., ApJ 560, (2001) L143

[2] Beloborodov, A. M., Illarionov, A. F., Ivanov, P. B., Polnarev, A. G., MNRAS 259, (1992) 209

[3] Carter, B., Luminet, J. P., A\&A 121, (1983) 97

[4] Carter, B., Luminet, J. P., MNRAS 212, (1985) 23

[5] Diener, P., Frolov, V. P., Khokhlov, A. M., Novikov, I. D., Pethick, C. J., ApJ 479, (1997) 164

[6] Guillochon, J., Ramirez-Ruiz, E., ApJ (submitted), (2012) arxiv: 1206.2350

[7] Hills, J. G., Nature 254, (1975) 295

[8] Ivanov, P. B., Novikov, I. D., ApJ 549, (2001) 467

[9] Ivanov, P. B., Chernyakova, M. A., Novikov, I. D., MNRAS 338, (2003) 147

[10] Ivanov, P. B., Chernyakova, M. A., A\&A 448, (2006) 843

[11] Kesden, M., Phys. Rev. D 85, (2012) 024037

[12] Zalamea, I., Menou, K., Beloborodov, A. M., MNRAS 409, (2010) L25 